\title{
RESPONSABILIDAD SOCIAL Y DISCAPACIDAD INTELECTUAL
}

\section{RESPONSIBILITY AND INTELLECTUAL DISABILITY}

\author{
David CRowther DCrowther@dmu.ac.uk \\ Centro de investigación en Gobernanza Organizacional. España. \\ De Montfort University. Reino Unido.
}

Teresa Duarte-Atoche tduarte@us.es

Universidad de Sevilla. España.

\begin{abstract}
RESUMEN
Hay muchos aspectos de la responsabilidad social corporativa que llegan a manifestarse de diferentes formas en diferentes entornos, pero los derechos humanos son uno de los tres aspectos más importantes. Este trabajo comienza con una revisión de los derechos humanos y de su importancia en los entornos empresariales antes de considerar los matices de la discapacidad que son afectados por la Responsabilidad Social Corporativa. Este artículo centra su atención principalmente en la discapacidad intelectual que a menudo se trata como un tema invisible y se elimina del discurso y de cualquier consideración en la estrategia corporativa. Argumentamos que los beneficios de incorporar una preocupación por la discapacidad intelectual en la planificación corporativa pesan más que las desventajas, no sólo en términos sociales y de capital humano sino también en términos financieros. Concluimos afirmando que la incorporación de este aspecto en la planificación corporativa es esencial para desarrollar cualquier negocio sostenible.
\end{abstract}

\section{Palabras Clave}

Discapacidad; Discapacidad intelectual; Responsabilidad Social Corporativa; Sostenibilidad.

\begin{abstract}
There are many aspects of corporate social responsibility which become manifest in different ways in different environments but human rights remains one of the 3 most important aspects. This paper commences with a review of this field and of the importance of human rights in the corporate environment before developing into a consideration of the aspects of disability which are affected by corporate social responsibility. Attention is focused primarily upon intellectual disability which is often treated as an invisible topic and eliminated from the discourse and from any consideration in corporate strategy. In this paper we explore this subject and argue that the benefits of incorporating a concern for intellectual disability into corporate planning outweigh the disadvantages, not just in human capital and social terms but also in financial terms. Furthermore we argue that incorporating this into corporate planning is essential in order to develop any sustainable business.
\end{abstract}

\section{KEYWORDS}

Corporate social responsibility; Disability; Intellectual disability; Sustainability. 


\section{INTRODUCCIÓN}

Se ha producido un cambio considerable en la percepción de la responsabilidad social corporativa (RSC) en los últimos tiempos. Parece que ha llegado a ser generalmente aceptado, por las empresas y sus directivos, por los gobiernos y sus agencias, y por el público en general, que existe un beneficio considerable en involucrarse con la responsabilidad social corporativa. Por consiguiente, cada vez más, las organizaciones están desarrollando sus políticas de RSC que están siendo integradas en la actividad empresarial. A pesar de que muchas personas siguen siendo escépticas sobre la legitimidad de estas actividades empresariales, la evidencia continúa señalando que las empresas están en realidad involucrándose en tales actividades socialmente responsables ${ }^{1}$; concretamente porque reconocen los beneficios que les reporta. Por tanto, parece que la batalla está ganada y todo el mundo acepta la necesidad de la actividad de RSC - todo lo que queda por discutir es cómo exactamente involucrarse en tal actividad y la manera de informar sobre ella-. Incluso esto ha sido ampliamente tratado a través de vehículos como el Global Reporting Initiative (GRI) y la ISO26000.

Sin embargo, la necesidad de la responsabilidad social no es de ninguna manera universalmente aceptada, pero la evidencia muestra que una serie de grandes empresas se están involucrando en el comportamiento ético y socialmente responsable con éxito -y este número sigue aumentando. Además no ha sido testado empíricamente que los rendimientos, en términos de rentabilidad, y la creación de valor, de las empresas que están involucradas en un comportamiento socialmente responsable, sean peores que los obtenidos por otras empresas que no lo están. De hecho, la proliferación de evidencia (ver Crowther 2002 para analizar las evidencias detalladas) pone de manifiesto que el comportamiento socialmente responsable conduce a un mayor rendimiento económico -al menos a largo plazo- y consecuentemente a un mayor bienestar y riqueza para todos los involucrados. Como corrobora el estudio realizado por Beurden y Gössling (2008), que testa la existencia de una relación positiva y significativa entre la actividad social empresarial y los beneficios empresariales. Su investigación la llevaron a cabo mediante un meta-análisis en el que estudiaron trabajos desde 1990. Esta técnica le permitió obtener como resultado que $23(68 \%)$ estudios determinaron que la relación entre la actividad social empresarial y el beneficio empresarial es positiva (Carter, Kale y Grima, 2000; Ruf, Muralidhar, Brown, Janney y Paul, 2001; Dowell, 2002; Kumar, Lamb y Wokutch, 2002; Goll y Rasheed, 2004; Schnietz y Epstein, 2005; Barnett y Solomon, 2006; Luo y Bhattacharya, 2006; He, Tian y Chen, 2007; entre otros) mientras que $9(26 \%)$ estudios

\footnotetext{
${ }^{1}$ La inversión socialmente responsable ha pasado a ser un inversión clave para inversores institucionales, por ello numerosas empresas trabajan en políticas socialmente responsables con el propósito de entrar en los índices bursátiles elaborados con criterios de RSC, tales como: Domini 400 Social Index, FTSE 4 Good, Citizens Index, Dow Jones Sustainability Group Index, ASPI (ARESE Sustainable Performance Indices), Calvert Social Index, Ethibel Sustainability, entre otros.
} 
revelaron que la relación no es significativa (McWilliams y Siegel, 2000; Moore, 2001; Seifert, Morris y Bartkus, 2003; Van de Velde, Vermeir y Corten, 2005; entre otros) , tan solo 2 (6\%) estudios concluyeron que no existe tal relación (Boyle, Higgins y Rhee, 1997; Brammer, Brooks y Pavelin, 2006).

Todo esto significa que hay una amplia variedad de actividades clasificadas como responsables socialmente, que van desde el altruismo hasta la triple bottom-line: performance financiera, social y medioambiental, y que han sido adoptados diferentes enfoques en diferentes países, en diferentes sectores e incluso en empresas diferentes aunque similares. Y, la tendencia actual está cambiando desde una preocupación por la responsabilidad social corporativa hacia una preocupación por la sostenibilidad; y muchas actividades han sido reasignadas en consecuencia (Aras y Crowther 2009a).

Esta preocupación por la sostenibilidad es interpretada muy a menudo como una preocupación por cuestiones medioambientales: estas son, por supuesto, muy importantes, pero sólo un aspecto de la sostenibilidad. Una segunda preocupación es normalmente económica o financiera, que también es importante. El aspecto social es frecuentemente descuidado y tratado como el menos importante de los tres pilares de la sostenibilidad. Este es el problema y el objetivo de este trabajo es examinar de nuevo este concepto de la sostenibilidad y lo haremos a favor de un nuevo y completamente diferente triple bottom-line que reconoce la importancia de las personas. Una vez analizado lo anterior, examinaremos la discapacidad intelectual y cuál es su lugar dentro de la responsabilidad social y la sostenibilidad. Para ello debemos comenzar con Brundtland y su legado.

\section{EL INFORME BRUNDTLAND Y DESPUÉS...}

En la actualidad, el término sostenibilidad es a la vez universal y un tema controvertido, ya que tiene significados diferentes para distintas personas. Sin embargo, existe una creciente sensibilización de que este es un tema de vital importancia y está siendo abordado por todas las organizaciones de una forma u otra (Comisiones Mundiales sobre el Medio Ambiente y Desarrollo (WCED) 1987; Schmidheiny 1992). El punto de partida debe ser establecido en el Informe Brundtland (WCED 1987), ya que hay una aceptación general de los contenidos de dicho Informe y porque la definición de sostenibilidad que enuncia es acertada y ampliamente aceptada. Igualmente, el Informe Brundtland es parte de un panorama político -especialmente aquel relacionado en la actualidad ${ }^{2}$ con el cambio climático- de los Estados y sus agencias, las grandes empresas supranacionales, así como las Naciones Unidas lo están debatiendo a través de vehículos como

\footnotetext{
${ }^{2}$ La preocupación en torno al cambio climático también está siendo tratado aunque con menor intensidad sobre tales cuestiones como población, migraciones, reducción de recursos, etc... El centro de las prioridades en la agenda política continúa cambiando de acuerdo con las circunstancias y la conveniencia.
} 
el WBCSD ${ }^{3}$ y la $C P{ }^{4}$, (véase, por ejemplo, Beder 1997; Gray y Bebbington 2001). La preocupación por el efecto que las medidas adoptadas en la actualidad tienen sobre las opciones disponibles en el futuro ha llevado directamente a la hipótesis simplista de que el desarrollo sostenible es deseable y posible, y una empresa puede demostrar la sostenibilidad simplemente por seguir existiendo en el futuro (Aras y Crowther 2008a). Por ello es importante recordar (WCED 1987: 1) la definición de desarrollo sostenible de la Comisión Brundtland que es la más aceptada y utilizada como definición estándar de desarrollo sostenible:

"... desarrollo que satisface las necesidades actuales sin comprometer la capacidad de las generaciones futuras para satisfacer sus propias necesidades".

Este Informe formula recomendaciones institucionales y legales para el cambio con el fin de hacer frente a problemas globales comunes. Cada vez más, hay un creciente consenso acerca de que las empresas y los gobiernos en asociación deben aceptar la responsabilidad moral por el bienestar social y para promover el interés de los individuos en las transacciones económicas (Murray, Haynes y Hudson 2010; Hudson 2009; Amba-Rao 1993).

Sin embargo, el Informe Bruntland hizo, de una manera significativa, una suposición -que nunca ha sido cuestionada, sino que simplemente se aceptó desde entoncessobre que el desarrollo sostenible era posible y deseable, y el posterior debate se ha centrado en cómo lograr esto. Así, desde que el Informe Brundtland fue elaborado por la Comisión Mundial sobre Medio Ambiente y el Desarrollo en 1987 ha habido un continuo debate sobre el desarrollo sostenible (Chambers 1994, Pretty 1995). Del mismo modo se ha hecho hincapié en aspectos tales como la colaboración, asociaciones y la participación de los interesados (Ladkin y Bertramini, 2002; Brown, Tompkins y Adger 2002). Sin embargo, ha sido generalmente aceptado que el desarrollo es deseable y que el desarrollo sostenible es posible - con un enfoque concomitante en la forma de lograr esto- . Sin embargo, el significado exacto de desarrollo sostenible ha estado mucho menos claro, y un punto de partida para cualquier análisis debe considerar exactamente qué se entiende por estos términos.

Hay un alto grado de confusión en torno al concepto de sostenibilidad: para la corriente más purista la sostenibilidad no implica nada más que la estasis — la capacidad de continuar de una manera sin cambios- pero a menudo se da a entender el desarrollo de manera sostenible (Marsden 2000; Hart \& Milstein 2003) y para muchos los términos de sostenibilidad y desarrollo sostenible son considerados sinónimos. Otros, sin embargo relacionan la sostenibilidad con el retorno de la mítica edad de oro de la

\footnotetext{
${ }^{3}$ World Business Council for Sustainable Development (Consejo de Negocios del Mundo por el DesarroIlo Sostenible).

${ }^{4}$ International Chamber of Commerce (Cámara de Comercio Internacional).
} 
autosuficiencia y la armonía con la naturaleza ${ }^{5}$. Nosotros tomamos la definición relacionada con la estasis (Aras y Crowther 2008b); a nivel corporativo, si el desarrollo es posible sin poner en peligro dicha estasis, entonces es un plus más que un componente de esta sostenibilidad. Además, el desarrollo sostenible es a menudo malinterpretado como centrarse exclusivamente en cuestiones medioambientales. En realidad, se trata de un concepto mucho más amplio, ya que las políticas de desarrollo sostenible abarcan tres áreas de política general: económica, medioambiental y social. En apoyo a esto, varios textos de las Naciones Unidas; y más recientemente, el Documento de Resultados de la Cumbre Mundial de 2005, que hace referencia a los "pilares interdependientes que se refuerzan mutuamente" del desarrollo sostenible como el desarrollo económico, desarrollo social y la protección del medio ambiente ${ }^{6}$.

\section{BRUNDTLAND Y SOSTENIBILIDAD}

A pesar de que este concepto tiene una antigüedad de más de 20 años el punto de partida debe ser el Informe Brundtland - sus definiciones han sido universalmente aceptadas. La sostenibilidad tiene que ver con el efecto que las medidas adoptadas en el presente tienen sobre las opciones disponibles en el futuro. Y, por supuesto, si los recursos se utilizan en el presente, entonces ya no estarán disponibles para su uso en el futuro.

El problema con Brundtland es que su preocupación por el efecto que las medidas adoptadas en el presente tiene sobre las opciones disponibles en el futuro nos ha llevado directamente a la enunciación de hipótesis simplistas como que el desarrollo sostenible es deseable y posible, y que las empresas pueden demostrar la sostenibilidad simplemente por seguir existiendo en el futuro (Aras \& Crowther 2008c). También ha llevado a la aceptación de lo que debe ser descrito como los mitos de la sostenibilidad: (i) La sostenibilidad es sinónimo de desarrollo sostenible y (ii) una empresa sostenible existirá simplemente por el reconocimiento de los problemas ambientales y sociales y su incorporación en su planificación estratégica.

Ambos se basan en una aceptación incondicional de la economía de mercado atribuida a la necesidad de crecimiento y en la falsa premisa de Brundtland a la que volveremos más adelante. Un supuesto casi incuestionable es que el crecimiento sigue siendo posible (Elliott 2005) y por lo tanto, la sostenibilidad y el desarrollo sostenible son sinónimos. De hecho, la perspectiva económica de las ontologías post-Cartesiano predomina y el crecimiento se considera que no sólo es posible sino también deseable

\footnotetext{
${ }^{5}$ Este enfoque hace caso omiso tanto al hecho de que los humanos han estado cambiando la naturaleza los últimos 10.000 años con la destrucción de los bosques y la extinción de especies animales, como, además, para la mayoría de la gente la existencia de la autosuficiencia ha sido siempre "ruda y corta" (Hobbes, 1651).

${ }^{6}$ En otras palabras el desarrollo social es un aspecto igual y esencial del desarrollo sostenible.
} 
(ver Spangenberg 2004). Así que es posible, por lo tanto, para Daly (1992) argumentar que la economía del desarrollo es lo único que debe ser tratado y puede serlo a través del mercado mediante la clara separación de los tres objetivos económicos básicos tales como la asignación eficiente, la distribución equitativa, y la escala sostenible. Hart (1997) va más allá y se refiere al concepto de desarrollo sostenible simplemente como una oportunidad de negocio, argumentando que, una vez que una empresa identifica su estrategia medioambiental, entonces las oportunidades de nuevos productos y servicios se hacen evidentes.

Al mismo tiempo todas las empresas están cada vez más preocupadas por su propia sostenibilidad y lo que el término realmente significa. Tal sostenibilidad es más que sostenibilidad ambiental. En cuanto a la sostenibilidad corporativa se refiere, la confusión se agrava por el hecho de que el término de desarrollo sostenible se ha utilizado en la literatura de gestión en los últimos 30 años (véase Reed y DeFillippi 1990) para suponer simplemente continuidad. Así Zwetsloot (2003) es capaz de combinar la responsabilidad social corporativa con las técnicas de mejora continua e innovación que implican que la sostenibilidad está de este modo asegurada. En consecuencia, la trayectoria de todos estos efectos está cada vez más centrada en el mismo tema.

En la actualidad, uno de los términos más usados en relación con la actividad empresarial es sostenibilidad. De hecho, puede afirmarse que ha sido usado en exceso, y aplicado con demasiados significados diferentes, por lo que efectivamente ha perdido sentido. Por tanto, es hora de volver a examinar el legado de Bruntland y redefinir lo que se entiende por actividad sostenible. Así, argumentamos que el desarrollo sostenible ha adquirido una importancia tal en el léxico de la conducta empresarial que es de hecho un imperativo estratégico, pese a haber escasa comprensión del término y de sus implicaciones (ver Aras \& Crowther, 2008b). Es parte de nuestro argumento que el uso actual del término, muy extendido por estar de moda, ha ofuscado cualquier comprensión real de la sostenibilidad. Esto es muy desafortunado, ya que consideramos que la sostenibilidad debe ser una parte integral del desarrollo estratégico de una empresa, pero es necesario un completo conocimiento de la sostenibilidad antes de que el desarrollo sostenible pueda ser tolerado.

La sostenibilidad es, por supuesto, fundamental para una empresa y su continuidad. Es igualmente fundamental no sólo para la continuidad de la actividad económica actual, sino también del propio planeta -al menos en una forma que entendemos actualmente. Es un proceso complejo, como ya lo hemos tratado. Además, es un proceso que debe reconocer no sólo la toma de decisión en la actividad operativa de la organización sino también las decisiones de distribución que se toman. Sólo entonces una organización será considerada sostenible.

Otros han asumido que una empresa sostenible existirá sólo por el reconocimiento de los problemas ambientales y sociales y su incorporación en su planificación estratégica. De acuerdo con Marrewijk y Were (2003) no existe una definición específica de la sostenibilidad corporativa, y cada organización tiene que diseñar su propia definición para que se ajuste a sus propósitos y objetivos, a pesar de que parecen asumir que la sostenibilidad empresarial y responsabilidad social corporativa son sinónimos y se basan en la actividad voluntaria que incluye inquietudes sociales y medioambientales. 


\section{LOS DESCENDIENTES DE BRUNDTLAND}

A partir del Informe Brundtland se han desarrollado distintos conceptos, incluyendo el de la triple bottom-line (Aras \& Crowther, 2008d). Esto a su vez ha llevado a una suposición de que abordar los tres aspectos de los derechos económicos, sociales y medioambientales es lo único que se necesita para garantizar no sólo la sostenibilidad, sino también permitir el desarrollo sostenible. De hecho, la asunción implícita es uno de los ejercicios más habituales, es decir, añadir un poco de información sobre los resultados medioambientales y sociales al informe financiero convencional y eso equivale al informe de la triple bottom-line. De esta forma, todas las empresas dan a entender que han reconocido los problemas, abordado las cuestiones a tratar y, con ello, asegurado el desarrollo sostenible. Esta implicación es generalmente aceptada sin ser cuestionada o sometida a ningún tipo de interrogatorio riguroso. Por ello, es necesario comenzar con un análisis de la triple bottom-line:

Figura 1.

La triple bottom-line

\begin{tabular}{ccc}
\hline & Sostenibilidad & \\
\hline Performance financiera & Performance medioambiental & Performance social \\
\hline
\end{tabular}

Fuente: elaboración propia

Abogamos por un rechazo de este concepto de la triple bottom-line por no estar suficientemente pulido para su uso práctico. Nuestro argumento es que el problema de la sostenibilidad ni siquiera se entiende, y mucho menos, se aborda correctamente. Además, los conceptos asociados con la triple bottom-line y con la idea del desarrollo sostenible no sólo son incorrectos, sino también realmente engañosos (véase Aras \& Crowther, 2008a) debido a la confusión existente en torno a los temas clave. Por tanto, es hora de volver a examinar el legado de Bruntland y redefinir lo que se entiende por actividad sostenible. Con el fin de hacer esto rechazamos los términos aceptados de la sostenibilidad y del desarrollo sostenible, prefiriendo el término de durabilidad para hacer hincapié en el cambio de enfoque. A partir de esto, abogamos por el rechazo de la triple bottom-line por no estar suficientemente depurada para su uso práctico y sugerimos en su lugar un modelo alternativo de la triple bottom-line real.

Obviamente a fin de lograr el desarrollo sostenible ${ }^{7}$ es necesario en primer lugar alcanzar la sostenibilidad, que a su vez depende de una serie de elementos. Por tanto, para con-

\footnotetext{
${ }^{7}$ Muchos autores continúan asumiendo tanto la posibilidad como la deseabilidad del desarrollo sostenible, como ya hemos mencionado anteriormente. Para nosotros, sin embargo, el logro de la sostenibilidad es una precondición necesaria y suficiente por sí misma.
} 
seguir la sostenibilidad no sólo es importante abordar cada elemento individualmente, sino también mantener el equilibrio entre ellos. El mantenimiento de este equilibrio es el aspecto más exigente y esencial de la gestión de la sostenibilidad. Hay una serie de elementos que deben ser abordados, pero estos se pueden agrupar en cuatro, que se asignan exactamente al modelo de evaluación de la sostenibilidad señalado anteriormente. Estos cuatro factores principales de la sostenibilidad (Aras \& Crowther, 2009b) son:

- Mantener la actividad económica, que debe ser la razón central de toda actividad empresarial y la razón principal para la organización de dicha actividad empresarial. Este elemento es la performance financiera.

- Conservación del medio ambiente, que es esencial para mantener las opciones disponibles para las generaciones futuras. Este es la performance del impacto medioambiental.

- Asegurar la justicia social, que incluye actividades tales como eliminar la pobreza, garantizar los derechos humanos, promover la educación universal y velar por la paz mundial. Este es la performance relativa a la influencia de la sociedad.

- El desarrollo de los valores espirituales y culturales, donde los valores corporativos y sociales se alinean en el individuo y gracias a este factor los demás performance son promovidos o negados; por desgracia en la actualidad en su mayoría son negados.

Nosotros argumentamos que la sostenibilidad se basa en abordar todos estos aspectos y que esto no se puede hacer simplemente a través de la promulgación de los contratos, ni a través de que la firma sea un nexo de los tratados (Williamson, 1975). No sólo son de vital importancia estos contratos negociados, sino también el contrato psicológico que comprende una parte esencial del Contrato Social ${ }^{8}$, así como proporcionar una base para el funcionamiento del contrato negociado. Y, por supuesto, esto sólo funciona en un ambiente de confianza - esto no puede ser escrito en el contrato y tampoco puede ser ignorado-. La confianza permite que las operaciones sean negociadas (Fukuyama, 1996). También permite a los contratos ser implementados, por ser lo suficientemente flexibles para permitir a la organización adaptarse a las circunstancias cambiantes. Es más, facilita la eliminación de las desigualdades de poder de la negociación y por lo tanto supera algunas de las deficiencias de la filosofía utilitaria que sustenta el sistema de mercado. Ha habido una tendencia a reclamar la racionalidad a través de la negociación fuera de cualquier contrato y nosotros argumentamos que esto sólo conduce a la eliminación de la sostenibilidad.

\footnotetext{
${ }^{8}$ En 1762 Jean-Jacques Rousseau publicó su libro sobre el Contrato Social que fue diseñado para explicar -y por lo tanto legitimar- la relación entre el individuo y la sociedad y su gobierno. Él argumentó que los individuos voluntariamente renuncian a ciertos derechos con el fin de que el Gobierno sea capaz de gestionar mejor los bienes para toda la población.
} 


\section{CONTABILIDAD Y ADMINISTRACIÓN}

La sostenibilidad implica una buena performance organizacional y un elemento clave de la buena performance corporativa es el de la administración y, por lo tanto, al igual que la gestión de una organización se ocupa de la administración de sus recursos financieros, también lo haría de la administración de los recursos medioambientales. La diferencia, sin embargo es que los recursos medioambientales están, en su mayoría, localizados fuera de la organización. La administración, en este contexto, por lo tanto está en relación con los recursos de la sociedad, así como con los recursos de la organización. La administración de recursos externos del medioambiente se desarrolla en base al principio central que garantiza la sostenibilidad. La sostenibilidad se centra en el futuro y se preocupa por hacer que las decisiones de utilización de recursos en el futuro no se vean limitadas por las decisiones tomadas en el presente. Esto necesariamente implica conceptos tales como la generación y utilización de los recursos renovables, reduciendo al mínimo la contaminación y usando nuevas técnicas de fabricación y distribución. También implica la aceptación de los gastos que puedan surgir en el presente como una inversión para el futuro.

Sin embargo, la actividad sostenible no sólo tiene impacto sobre la sociedad en el futuro, sino también sobre la propia organización en el futuro. Por lo tanto, el buen desempeño medioambiental de una organización en el presente es en realidad una inversión en el futuro de la propia organización. Esto se logra a través de la garantía de los suministros y las técnicas de producción que permitirá a la organización operar en el futuro de una forma similar al presente y así emprender una actividad de creación de valor en el futuro de igual forma que lo hace en el presente. No obstante, la gestión financiera también se preocupa de la gestión de los recursos de la organización en el presente para que la organización cree valor en el futuro. Por lo tanto la gestión interna de la empresa, desde una perspectiva financiera, y su gestión medioambiental externa coinciden en esta preocupación común para la gestión del futuro. La buena performance financiera conduce a un buen desempeño futuro en la dimensión medioambiental y viceversa. Por lo tanto, no existe una dicotomía (Crowther 2002) entre el desempeño medioambiental y el financiero, sino que los dos aspectos se confunden en una sola preocupación. Esta preocupación es, por supuesto, la gestión del futuro en cuanto a la empresa a que se refiere 9 . El papel de la contabilidad social y medioambiental y el de la contabilidad e información financiera por lo tanto, pueden ser vistos como fortuitos. De este modo, el trabajo requerido para lograr la sostenibilidad no está en torno a la distribución de recursos, sino más bien al desarrollo de medidas que reflejen verdaderamente las actividades de la organización en su entorno.

${ }^{9}$ Los informes financieros están basados, por supuesto, en la continuidad de la empresa —el principio del negocio en funcionamiento- 
Estas técnicas de medición y, en consecuencia los informes, es un precursor necesario para tratar la gestión del futuro y por lo tanto la sostenibilidad.

Del mismo modo la creación de valor en una empresa es medida a través de las ganancias obtenidas por los actores de dicha empresa, tales como los accionistas u otros. Sin embargo, el valor debe ser tomado en su definición más amplia para incluir otros valores además del económico, ya que es posible que el valor económico pueda ser creado a expensas de otros componentes del bienestar, como el bienestar espiritual 0 emocional ${ }^{10}$. Esta creación de valor por la empresa añade bienestar para la sociedad en general, aunque este bienestar está dirigido a determinados miembros de la sociedad en lugar de tratar a todos como iguales. Esto ha llevado a los argumentos de Herremans et al. (1992), Gray (1992) y Tinker (1988), entre otros, relacionados con la distribución del valor creado y de si el valor es creado por un grupo de interesados, a expensas de los demás. No obstante, si al sumarse, el valor se crea, entonces esto se suma al bienestar de la sociedad en general, comoquiera que se distribuya. De forma similar, los buenos resultados medioambientales conducen a un mayor bienestar para la sociedad en general, aunque esto tenderá a ser expresado en términos emocionales y de la comunidad en lugar de ser capaz de expresarse en términos cuantitativos. Esto se expresa en una sensación de bienestar, que, por supuesto, conduce a una mayor motivación. Este aumento de la motivación conducirá inevitablemente a una mayor productividad, alguna parte de la cual beneficiará a las organizaciones, y también un deseo por mantener un ambiente agradable, que a su vez conducirá a un medioambiente mejorado, a una acentuación del bienestar y a la reducción de los aspectos destructivos del compromiso social por los individuos.

\section{ASPECTOS DE LA SOSTENIBILIDAD}

Hay una serie de cuestiones que actualmente son de interés para las empresas y personas (Crowther y Seifi, 2010). En términos generales se puede considerar que estas cuestiones son las relativas al medioambiente, la protección de los derechos humanos y la gobernabilidad. Podemos examinarlos de uno en uno.

\section{Cuestiones medioambientales}

Los cambios en los sistemas climáticos de todo el mundo son evidentes para la mayoría de la gente y se manifiestan en un clima tan extremo como el exceso de lluvia o nieve,

\footnotetext{
${ }^{10}$ Ver Crowther, Davies y Cooper (1998), Ormerod (1994) y Mishan (1967). Esto puede ser equiparado al concepto de utilidad del discurso sobre el liberalismo clásico.
} 
sequías, olas de calor y huracanes que han afectado a muchas partes del mundo. De hecho la mayoría de nosotros recordamos, por ejemplo, el huracán Katrina que devastó Nueva Orleans en 2005. Del mismo modo el problema con Eyjafjallajökull ${ }^{11}$ fue causado por el calentamiento global que derrite el glaciar que lo rodea. El calentamiento global y el cambio climático, el efecto más notable, es un tema de discusión en todo el mundo y por lo general, aunque no de una manera universal, ni percibido de la misma forma por todos los individuos (Moyano, Paniagua y Lafuente 2009: 683), es aceptado que el calentamiento global está ocurriendo y que seguirá transcurriendo. Sin embargo, la opinión está dividida acerca de si el cambio climático que ha tenido lugar se puede revertir o no. Algunos piensan que no, tal y como afirma Lovelock (2006) el cambio climático es inevitable, con consecuencias sobre el medioambiente y por lo tanto sobre la vida humana y la actividad económica.

Aunque hay muchos factores que están contribuyendo al calentamiento global que está teniendo lugar, es evidente que la actividad comercial y económica juega un papel importante. De hecho mucha gente habla de los "gases de efecto invernadero" siendo el dióxido de carbono la principal causa, como consecuencia directa de la actividad económica. Por lo tanto muchas personas ven la reducción de las emisiones de estos gases como algo fundamental para cualquier intento de combatir el cambio climático. Por supuesto, esto requiere un cambio en el comportamiento - de las personas y de las organizaciones-. Cohen et al. (1998) sugieren que los problemas ambientales ligados al cambio climático modifican los esquemas sobre las percepciones y actitudes ambientales de la población que han inspirado las políticas de desarrollo sostenible. Por tanto, percibida esa necesidad de cambio, ha provocado un aumento de la preocupación por la sostenibilidad que inspirará cambios en las políticas para combatir los problemas ambientales.

Otro factor que preocupa a la población en general es el de la huella ecológica -la cantidad de espacio físico de la tierra necesaria para abastecer a cada persona-. En el análisis de la huella ecológica se compara la demanda humana sobre la naturaleza con la capacidad de la biosfera para regenerar los recursos y la prestación de servicios. Para ello, se evalúa la tierra biológicamente productiva y el área marina necesaria para producir los recursos que una población consume, y para absorber los desechos correspondientes, utilizando la tecnología imperante. Este enfoque también puede aplicarse a una actividad como la fabricación de un producto o a la conducción de un coche. Un término que posiblemente está más de moda en la actualidad es la huella de carbono ${ }^{12}$.

\footnotetext{
${ }^{11}$ El volcán en Islandia interrumpió los vuelos en toda Europa en 2010.

${ }^{12}$ Esto convenientemente ha ofuscado el problema. El dióxido de carbono no es el único gas de efecto invernadero y no es el más problemático pero nos centramos en él porque el dióxido de carbono de la industria de vehículos de motor puede ser ignorado además del gas metano de la industria diaria. Estas industrias son también la causa de otros problemas medioambientales importantes.
} 
Para una persona la definición de la huella de carbono es la cantidad total de dióxido de carbono atribuible a las acciones de ese individuo (principalmente a través de su consumo de energía) durante un período de un año. Esta definición determina los calculadores personales de carbono que son ampliamente utilizados ${ }^{13}$. El término tiene su origen en la idea de que una huella es lo que se ha quedado atrás, como resultado de las actividades del individuo. Se pueden considerar como emisiones de carbono sólo las directas (por lo general a partir de la energía utilizada en el hogar y en el transporte, incluidos los viajes por los automóviles, aviones, trenes y otros medios de transporte), o también puede incluir las emisiones indirectas (incluyendo las emisiones de dióxido de carbono como resultado de los bienes y servicios de consumo). Normalmente se entiende que las emisiones de dióxido de carbono (y las emisiones de otros gases de efecto invernadero) se asocian casi exclusivamente con la conversión de los portadores de energía, tales como leña, gas natural, carbón y petróleo. El contenido de carbono liberado durante el proceso de conversión de la energía llega a la atmósfera y se considera responsable del calentamiento global, y por lo tanto del cambio climático ${ }^{14}$. Sin embargo, la preocupación general se ha expresado en todo el mundo y esto lo ha llevado al Protocolo de Kyoto ${ }^{15}$. El Protocolo de Kyoto define objetivos legalmente vinculantes y calendarios para reducir las emisiones de gases de efecto invernadero de los países industrializados que han ratificado el protocolo ${ }^{16}$.

A pesar de que la opinión científica más o menos haya llegado a un consenso sobre que el calentamiento global está ocurriendo y por lo tanto, también el cambio climático, todavía hay un considerable número de escépticos y de los que niegan que esté sucediendo ${ }^{17}$. Hay otros que argumentan que la contribución humana al calentamiento global es insignificante: por lo tanto, afirman que es inútil o incluso perjudicial concentrarse en las contribuciones individuales.

En muchas partes del mundo el agua se está convirtiendo en un problema serio. Concretamente, el riego ha provocado serios problemas en algunas partes del mundo como California, mientras que en Uzbekistán se ha reducido el mar de Aral a una pequeña fracción de su tamaño original ${ }^{18}$. Y de muchos ríos, en todas partes del mundo, se ha extraído tal cantidad de agua que ya no llegan al mar. Al mismo tiempo, millones de per-

\footnotetext{
${ }^{13}$ Herramienta desarrollada en 2007 por ADEME y el Clima Futures Association.

${ }^{14}$ Por supuesto, esto es muy simplista, si no completamente erróneo. La gente y animales producen dióxido de carbono cuando respiran; las vacas (y otros rumiantes) producen gas metano y el proceso mediante el que la vegetación produce, captura y seguidamente emite dióxido de carbono es complejo y no totalmente comprendido (Lomborg 2001).

${ }^{15}$ Se acordó en 1997 y entró en vigor en 2005.

${ }^{16} \mathrm{~A}$ finales de 2007 Australia ratificó el protocolo, existiendo entonces sólo un gran país desarrollado no adherido. Este país es USA, probablemente el mayor emisor de gases de efecto invernadero.

${ }^{17} \mathrm{El}$ consenso europeo no quiere decir mundial a este respecto.

${ }^{18} \mathrm{El}$ Aral era el cuarto lago más grande del mundo (unos 66.000 kilómetros cuadrados), en 2004 ya había perdido un $75 \%$ de su extensión, y sus aguas habían quintuplicado su salinidad.
} 
sonas no tienen acceso al agua potable. Y los países están entrando en disputas por el acceso al agua que comparten. De hecho el acceso al agua se prevé que se convierta en una importante fuente de conflictos en el siglo XXI. Otra de las cuestiones relacionadas con el agua es el tema del agua virtual; la Real Academia de Ingeniería (Reino Unido) ha documentado (2010) que los países como el Reino Unido están utilizando el agua de los países en desarrollo donde hay escasez, mediante su incorporación en los productos comprados a dichos países.

Es bastante obvio que los recursos del planeta son finitos y este es un factor que limita el crecimiento y desarrollo. El agotamiento de los recursos del planeta sin embargo es uno de los factores que ha ayudado a crear el actual interés en torno a la sostenibilidad. De particular interés es el de las industrias extractivas y concretamente el aluminio cuyo suministro sólo está asegurado a corto plazo. En el Reino Unido los recursos minerales como el estaño y el plomo fueron completamente extraídos hace mucho tiempo y las prósperas industrias en torno a ellos desaparecieron. Al igual que ocurre con otros recursos - tales como el carbón- que se extraen en su totalidad, entonces las empresas basadas en dichos recursos desaparecen, al igual que los puestos de trabajo vinculados a estas industrias. Esta es una fuente de preocupación obvia para la gente.

De especial preocupación es la extinción del suministro de petróleo, porque una gran parte de la actividad económica sólo es posible con el uso de energía creada a partir del petróleo. De hecho, muchos sostienen que las guerras en el Medio Oriente ${ }^{19}$, en particular los problemas en Irak e Irán, han sido causadas por la escasez de petróleo, real o inminente, y los problemas que ello pueda causar, más que por cuestiones políticas. La mayoría de la gente ya ha oído hablar del Pico de Hubbert ${ }^{20}$ y están comprometidas con el debate sobre si se ha alcanzado o no (Bower 2009; Deffeyes 2004). Ciertamente se ha hecho en partes del mundo como EEUU y el Mar del Norte, pero no se ha alcanzado en todo el mundo. Sin embargo, el quid de la sostenibilidad -y del desarrollo sostenible- se basa en la necesidad de energía y no hay suficientes fuentes alternativas de energía para compensar la eliminación del petróleo como fuente de combustible. Por consiguiente el agotamiento de los recursos, real o imaginario, y en particular la energía, es una de las causas más importantes del interés actual en la sostenibilidad.

\footnotetext{
${ }^{19}$ Y la mayoría probablemente de cualquier otra parte del mundo también — sería instructivo para relacionar la presencia de petróleo con los conflictos.

${ }^{20}$ En 1956 M. King Hubbert desarrolló un modelo de producción de petróleo que mostró que cuando las reservas de petróleo llegaran al punto medio entonces la producción se ralentizaría y habría menos petróleo disponible. Aunque originalmente es un modelo desarrollado por la producción de petróleo en EE.UU, esto es igualmente válido a nivel global. Este punto medio es conocido como el Punto Hubbert y ha llegado o pronto llegará, en este punto los suministros de petróleo comenzarán a reducirse con obvias implicaciones en el medioambiente, en el que existe un crecimiento continuo de la demanda.
} 


\section{Cuestiones sobre derechos humanos.}

La responsabilidad de la empresa de respetar los derechos humanos es reconocida por prácticamente todas las iniciativas RSC de las empresas y las industrias, es respaldada por las principales asociaciones empresariales del mundo, se ha afirmado en el Pacto Mundial y en sus redes nacionales y mundiales y está enunciada en instrumentos jurídicos tales como la Declaración Tripartita de la OIT y las directrices de la OCDE, así que es una norma social acreditada e institucionalizada, que existe independientemente de los derechos de los Estados y las variaciones de la ley nacional. La responsabilidad de respetar los derechos humanos es una norma básica para todas las empresas en todas las situaciones (Human Rights Council 2009; citado como A/HRC/11/13 a partir de ahora).

Una cuestión en torno a los derechos humanos que preocupa a la sociedad está relacionada con la cadena de suministro de un negocio, es decir, con lo que ocurre en otras sociedades con las que la empresa hace negocios - sus proveedores y los proveedores de sus proveedores-. En particular, la gente está preocupada con la explotación de personas en los países en desarrollo, especialmente con la cuestión del trabajo infantil, aunque también con los talleres clandestinos.

Así que ya no es aceptable que una empresa diga que las condiciones en las que operan sus proveedores se encuentran fuera de su control y, por lo tanto, no son responsables. La violación de esta norma social suele darse a conocer en todo el mundo mediante la movilización de las comunidades locales, las redes de la sociedad civil, los medios de comunicación, con inclusión de los blogs, los procedimientos de reclamación como los PNC de la OCDE y, si están implícitas presuntas violaciones de la ley, posiblemente los tribunales (A/HRC/11/13). Estas prácticas desencadenan repercusiones muy negativas en las empresas que las llevan a cabo, viéndose afectada su actividad económica.

Recientemente ha habido una serie de compañías de alto perfil de venta al por menor que han alzado las manos para reconocer los problemas y luego han dado los pasos necesarios de forma pública para cambiar esto. Curiosamente la popularidad de las empresas aumenta después de haber admitido los problemas y tomado medidas para corregir estos problemas. Al hacer esto, muestra tanto que la honestidad es la mejor práctica como también que los clientes son razonables. La evidencia sugiere que los clientes individuales son comprensivos y que ellos no esperan la perfección, pero sí la honestidad y la transparencia. Por otra parte, también esperan que las compañias hagan esfuerzos para cambiar su comportamiento y tratar de resolver sus problemas de RSC.

Las empresas también han cambiado. Ya no se preocupan de publicidad ecológicoengañosa (greenwashing) -la pretensión de un comportamiento socialmente responsable a través de astutos informes-. Ahora las empresas se están tomando la RSC mucho más en serio, no sólo porque entienden que es clave para el éxito del negocio y les puede dar una ventaja estratégica, sino también porque a la gente en esas organizaciones le preocupa la responsabilidad social. 
Por lo que sería razonable afirmar que la creciente importancia de la RSC y la sostenibilidad están siendo impulsadas por las personas que se preocupan; pero esas personas no son sólo clientes, sino también empleados, gerentes, propietarios y los inversores de una empresa. Así, las empresas están reaccionando en parte a las presiones externas y, en parte, liderando el desarrollo de un comportamiento responsable y de la información.

\section{Discapacidad intelectual y derechos humanos.}

La discapacidad y su tratamiento es un tema muy importante para la sostenibilidad. «La teoría tradicional (...) sitúa la fuente de la discapacidad en la deficiencia del individuo y en sus discapacidades personales. En contraposición, el modelo social entiende la discapacidad como el resultado del fracaso de la sociedad al adaptarse a las necesidades de las personas discapacitadas» (Abberley, 1998: 78) ${ }^{21}$. Según Ferreira (2008: 155), la representación colectiva de la discapacidad no es la de una diferencia integrada en un mundo caracterizado por la diversidad —diversidad que efectivamente es condición propia de nuestra convivencia-, sino que indica, muy al contrario, la existencia de una deficiencia que se traduce en inferioridad respecto de un hipotético estándar de normalidad: discapacidad es minus-valía; como alternativa a esa evidente catalogación social de la discapacidad, pues lo que efectivamente vive la persona con discapacidad es otro modo de valerse en ese entorno existente, encontramos el concepto de Diversidad Funcional22.

A pesar de los esfuerzos realizados por investigadores ${ }^{23}$, sistemas políticos y el propio colectivo de discapacitados vivimos en una sociedad en la que las personas con discapacidad están en desventaja: son discriminadas, tal y como afirma Ferreira (2008).

Según la clasificación internacional del funcionamiento, de la discapacidad y la salud (CIF) realizado por la Organización Mundial de la Salud (OMS) en 2001, existen diversas discapacidades y entre ellas encontramos la discapacidad intelectual, que supone el $59,4 \%$ de las personas con discapacidad (Instituto Nacional de Estadística 2008), es decir, es el motivo de discapacidad más destacado. Como ya hemos señalado antes, los discapacitados intelectualmente han sido excluidos por ser diferentes, es más, eran encerrados donde no se les viera. Así, por ejemplo los enfermos mentales o discapacitados en Ingla-

\footnotetext{
${ }^{21}$ Rodríguez y Ferreira (2010) realizan un análisis muy interesante sobre la evolución del concepto de discapacidad (desde el modelo médico-rehabilitador, pasando por el modelo social-emancipatorio, hasta llegar a la diversidad funcional).

${ }^{22}$ Este nuevo concepto surge en 2005 promovido por el movimiento español, por una Vida Independiente, que creó en Internet el Foro de Vida Independiente (FVI) en 2001. Página web del FVI: http://es.groups. yahoo.com/group/vidaindependiente.

${ }^{23}$ En España la investigación en torno a la discapacidad es muy escasa (véase en Ferreira 2008: 143), por el contrario encontramos numerosísimos trabajos cientíícos, a la vez que pioneros en el mundo anglosajón (véase, por ejemplo, http://www.disabilityarchive.leeds.ac.uk/).
} 
terra fueron encerrados en Bedlam ${ }^{24}$ a partir del siglo XIV. Esta práctica fue seguida en el Occidente hasta la última parte del siglo XX. Sin embargo, en el Imperio Otomano las diferencias mentales fueron tratadas como una enfermedad que se curaba ${ }^{25} \mathrm{y}$ los tratamientos en el siglo XVI no se conocieron en Occidente hasta mediados del siglo XX.

Más recientemente, se ha aceptado de forma general que la discapacidad no debe ser motivo para impedir el pleno acceso a los derechos humanos y la participación plena en la sociedad. Así, por ejemplo la Declaración de Montreal sobre la Discapacidad Intelectual ${ }^{26}$ afirma esto en nombre de todas las personas. Del mismo modo la UNESCO ha elaborado un conjunto de principios en que se basa el tratamiento de las personas con discapacidad y en la misma línea ha trabajado La Agencia Europea de Derechos Fundamentales. Todos los principios esbozados por los distintos organismos se parecen en que pretenden lograr la integración de las personas en la sociedad en lugar de separarlos -la asimilación y la inclusión en lugar de la discriminación y la exclusión. No obstante, es necesario que se produzcan cambios importantes para que el entorno social sea realmente integrador con respecto a la discapacidad.

En Occidente este enfoque integrador es reciente y bienvenido. El tratamiento equitativo es un ingrediente esencial de la sostenibilidad. Para una empresa, por supuesto, también tiene buen sentido económico ser más inclusivos: el capital humano se traduce en otro capital. De acuerdo con Bourdieu (1984) hay tres tipos de capital que cada uno de nosotros poseemos: el capital social, capital cultural y capital económico.

Al nivel más simple, el capital social se refiere a nuestra capacidad para comportarnos adecuadamente en las circunstancias y la sociedad en la que nos encontramos. Así, tenderemos a comportarnos de manera diferente en diferentes circunstancias, tales como asistir a una cena formal, estar presente en una reunión de trabajo o ver un partido de fútbol con nuestros amigos, y probablemente hemos visto personas que se han comportado de manera inapropiada para las circunstancias en las que se encuentran. En términos generales cuantas más circunstancias haya, más estamos en condiciones de actuar en consecuencia, mayor es la cantidad de capital social que poseemos. El capital

\footnotetext{
${ }^{24}$ Bedlam es el hospital Real de Bethlehem en Londres que también representa el paradigma de la discapacidad intelectual.

${ }^{25}$ Mientras que en los países occidentales durante la Edad Media los enfermos mentales fueron sometidos a severas torturas inhumanas e incluso asesinados porque el diablo poseía su espíritu, en DARÜŞŞIFA (hospital psiquiátrico), sección de la institución en Edirne que fue promovido por el sultán Bayezit II y construido por el arquitecto Hayrettin en 1488, los enfermos mentales eran tratados con LA MÚSICA. EVLIYA ÇELEBI, quien visitó DARÜŞŞIFA en 1640, explicó en su libro de viajes la forma en que los enfermos mentales eran tratados con la música. El tratamiento con la música no era de hecho un invento de los médicos turcos otomanos. Sin embargo, los médicos turcos otomanos, que alcanzaron un nivel superior a sus contemporáneos en las prácticas de salud mental a través de sus estudios científicos, estaban muy aventajados en el tratamiento de pacientes con la música. http://turkeytourism.bayanposta.com/wiki/ Turkish_History_of_Psychiatry_Museum__Edirne

${ }^{26} \mathrm{http}: / /$ www.mdri.org/mdri-web-2007/pdf/montrealdeclaration.pdf
} 
social procede inicialmente de nuestra familia y nuestra educación, pero también se adquiere mediante la experiencia y la práctica $-\mathrm{y}$ cuanto más se posee mayor es la variedad de situaciones en las que nos sentimos cómodos.

El capital cultural se refiere a la variedad de personas que conocemos y con quienes tenemos contacto. En esencia, el capital cultural, por tanto, equivale a nuestra red social. Cuanto más grande es nuestra red, sobre todo cuando contiene una amplia gama de personas de diferentes orígenes, mayor es la cantidad de capital cultural que poseemos. Si sabemos que podemos interactuar con mucha gente, $y$ todas estas personas se conocen entre sí, entonces la red social de cada una de ellas coincide en gran medida y esto no nos proporciona mucho capital social. Se trata de conocer personas que tienen diferentes redes, pero sobre todo no se solapen, que nos ofrezcan su capital cultural porque esto nos permite ampliar nuestra propia red social. También es siempre importante recordar que cuanto mayor es la influencia -en cualquier término o esfera que sean importantes para nosotros- de la gente en nuestra red social, mayor es entonces el capital cultural que esta nos facilita. Nuestro capital cultural procede de nuestra capacidad para usar nuestra red social de manera que sea beneficioso para nosotros. La posesión de una red social, y por lo tanto del capital cultural, no es, por supuesto, solamente para utilizarlo en nuestro propio beneficio, ya que los demás también están tratando de hacer uso de nuestra red para ayudarles. Así que, esencialmente una red social es un mecanismo mediante el cual las personas pueden ayudarse unas a otras para alcanzar sus metas y objetivos individuales. Esta asistencia mutua fortalece la red y al mismo tiempo aumenta la cantidad de capital cultural que nosotros, como individuos, poseemos.

Hasta cierto punto, el capital cultural también viene de nuestra familia y nuestra educación, pero también se adquiere, por ejemplo, en la universidad. Por esta razón los estudiantes de entornos ricos e influyentes empiezan sus vidas con una ventaja -tienen mayor capital cultural que pueden utilizar a lo largo de sus vidas. Y las universidades de mayor prestigio —a las que suelen ir los que ya poseen más capital cultural— extienden esa ventaja.

El capital económico se presenta de dos formas -la riqueza y los ingresos. A pesar de que la riqueza, en cualquiera de sus formas, se puede heredar, no ocurre lo mismo con los ingresos. Los ingresos se deben obtener por nuestros propios esfuerzos.

Es importante entender que los tres tipos de capital pueden convertirse en otros tipos de capital. Así, el capital social puede ser convertido en capital cultural, porque si entendemos cómo comportarnos adecuadamente en una amplia gama de circunstancias, entonces podemos ampliar nuestra red social y, por lo tanto, aumentar la cantidad de capital cultural que poseemos. Del mismo modo podemos hacer uso del capital cultural que tenemos para que nos brinde la oportunidad de un empleo más ventajoso 0 mejores oportunidades para obtener ingresos y riqueza. De esta manera, nuestro capital económico puede ser mayor (Crowther y Seifi 2012).

Las preguntas que nos planteamos tras el análisis del capital son las mismas que se hizo Brogna (2006: 1) “¿Por qué la mayor parte de las personas con discapacidad no 
están escolarizadas en escuelas comunes? ¿Por qué pasan años en escuelas especiales o talleres protegidos? ¿Por qué no son nuestros compañeros de facultad, de trabajo, por qué no se casan con nuestros hijos?" y Ferreira (2008: 161) "¿Podemos afirmar que la discapacidad es una variable que condiciona significativamente la posición de los individuos dentro de la estructura social?" y por tanto, ¿El capital social, cultural y económico ${ }^{27}$ que poseen y su capacidad para ampliarlos es muy limitada?. En el entorno social en el que vivimos las personas con discapacidad están en desventajas, sufren discriminación y exclusión, por tanto, soportan grandes dificultades para alcanzar, por sus propios esfuerzos, capital social, cultural y económico. Según la última encuesta realizada por el INE sobre discapacidad, autonomía personal y situaciones de dependencia (EDAD 2008) 3,85 millones de personas residentes en hogares afirman tener algún tipo de discapacidad o limitación -esto supone una tasa de 85,5 por mil habitantes, siendo la deficiencia que causa mayor número de discapacidades por persona la mental: 11,6 frente a las 8,7 de media que tienen las personas con discapacidad.

Si analizamos la actividad principal que desempeñan en su vida cotidiana las personas con discapacidad en edad de trabajar-entre 16 y 64 años- que suponen 1,48 millones de personas, se observa que el $41 \%$ recibía algún tipo de pensión, un $28,3 \%$ trabaja y un $7,2 \%$ estaba desempleado (véase tabla 1 ).

Tabla 1.

Personas con discapacidad de 16 a 64 años en función de su relación principal con la actividad

\begin{tabular}{l|c}
\hline & $N^{0}$ de personas (miles) \\
\hline TOTAL & $1.482,1$ \\
\hline ACTIVOS & 419,3 \\
Trabajando & 106,8 \\
En desempleo & \\
\hline INACTIVOS & 451,1 \\
Percibiendo pensión contributiva & 157,2 \\
Percibiendo otro tipo de pensión & 183,6 \\
Dedicado/a principalmente a labores del hogar & 87,2 \\
\hline Incapacitado/a para trabajar(sin recibir pensión) & 33,9 \\
Estudiando & 43,1 \\
Otros inactivos & \\
\hline
\end{tabular}

Fuente: INE (2008)

\footnotetext{
${ }^{27}$ Una persona con discapacidad que pertenece a una clase social alta disfrutará de los privilegios de dicho grupo, por tanto, poseerá capital económico, aunque las barreras para lograr el capital social y cultural será similares a las de cualquier otra persona con discapacidad que no pertenezca dicha clase social.
} 
El menor porcentaje de población ocupada estaba en los colectivos que tenían limitaciones de aprendizaje y aplicación de conocimientos y desarrollo de tareas (con un $8,2 \%)$.

Con respecto al nivel de estudios alcanzado por la población con discapacidad se observa importantes diferencias en los niveles inferiores de estudios, donde los porcentajes de personas discapacitadas que no saben leer, que tienen estudios primarios incompletos o completos son mayores que los que representa a la población total. Del mismo modo, el porcentaje de personas con discapacidad que tienen estudios universitarios son menores en casi 24 puntos con relación a la población total. Si comparamos la población con discapacidad intelectual con la población total discapacitada se observa una gran brecha, siendo las personas con discapacidad intelectual las que encuentran mayores obstáculos para alcanzar los distintos niveles de estudios (véase tabla 2)

Se pone de manifiesto que las personas con discapacidad, y mucho más acentuadamente con discapacidad intelectual, tienen dificultades para acceder al mercado laboral, para alcanzar los distintos niveles educativos... por tanto, en parte ${ }^{28}$ estos datos confirman que la discapacidad sí influye en la posición del individuo en la estructura social y por supuesto en su capital.

Tabla 2.

Nivel de estudios alcanzados por la población

(\% personas de 25 a 64 años)

\begin{tabular}{lccc}
\hline & $\begin{array}{c}\text { Total } \\
\text { Población } \\
(\%)\end{array}$ & $\begin{array}{c}\text { Población } \\
\text { discapacitada } \\
(\%)\end{array}$ & $\begin{array}{c}\text { Población con } \\
\text { discapacidad } \\
\text { Intelectual } \\
(\%)\end{array}$ \\
\hline No sabe leer o escribir & 0,9 & 8,6 & 21,3 \\
Estudios primarios incompletos & 2,1 & 11,5 & 37,8 \\
Estudios primarios o equivalentes & 7,7 & 23,3 & 22,8 \\
Educación secundaria de primera etapa & 29,0 & 19,2 & 6,3 \\
Estudios de bachillerato & 15,8 & 11,6 & 3,0 \\
\hline Enseñanzas profesionales de grado medio o equivalentes & 9,1 & 9,7 & 1,4 \\
Enseñanzas profesionales de grado superior o equivalentes & 11,4 & 5,6 & 0,8 \\
Estudios universitarios o equivalentes & 24,1 & 10,5 & 2,7 \\
\hline
\end{tabular}

Fuente: Encuesta de Población Activa (EPA) y elaboración propia

\footnotetext{
${ }^{28}$ Aunque como afirma Ferreira (2008: 162) sería necesaria una investigación en torno a la estratificación social.
} 
Creemos que la inclusión e integración de personas con discapacidad en general, e intelectual en particular, enriquecería este proceso, lográndose mejor performance en cuestiones de derechos humanos y por tanto, se estaría más cerca de lograr la sostenibilidad.

\section{Una crisis de gobernanza}

La crisis financiera y económica de 2008 ha demostrado que existen fallos en la gobernanza y problemas con el sistema de mercado. En general, estos han sido descritos como representantes de fallos sistémicos del sistema de mercado y la aplicación poco estricta de los sistemas de gobernanza y regulación. Por ello, se aboga por mejorar dichos sistemas con el fin de combatir esta crisis. Naturalmente, se ha hablado de estos fallos y los consiguientes problemas y se seguirá haciendo en el futuro. No es, por supuesto, la primera crisis y la economía de mercado se ha ido desarrollando sobre el curso de boom y quiebra durante los últimos 20 años, que no es muy diferente al de los años sesenta y setenta, que los neo-conservadores afirmaron haber detenido. Las principales diferencias son que los últimos ciclos han sido impulsados por los mercados financieros y que la era de la globalización significa que ningún país es inmune a los efectos sufridos en otros países.

Cuando estamos pensando en alternativas, tenemos por lo tanto que analizar profundamente el concepto de gobernanza. Todos los sistemas de gobierno se refieren principalmente a la gestión de la gobernanza de las asociaciones y por lo tanto, a la autoridad política, a las instituciones y, en última instancia, al control. La gobernanza en este sentido particular denota las instituciones políticas formales que tienen como objetivo coordinar y controlar las relaciones sociales interdependientes y que tienen la capacidad de hacer cumplir las decisiones. Cada vez más, sin embargo, en un mundo globalizado, el concepto de gobernanza ${ }^{29}$ se utiliza para describir la regulación de las relaciones de interdependencia en la ausencia de la autoridad política general, como en el sistema internacional. Así, la gobernanza global o "gobernanza sin gobierno" (Rosenau y Czempiel 1992) se puede considerar como la gestión de los procesos globales en la ausencia de forma de un gobierno global. Hay algunos organismos internacionales que tratan de abordar estas cuestiones y entre estos están las Naciones Unidas y la Organización Mundial del Comercio. Cada uno de estos ha tenido un éxito desigual en el establecimiento de alguna forma de gobernanza en las relaciones internacionales, pero forman parte del reconocimiento del problema y del intento de abordar los problemas mundiales que van más allá de la capacidad de los estados individuales para resolverlos.

Gobernanza global no es, por supuesto, lo mismo que un gobierno mundial: de hecho, se puede argumentar que tal sistema en realidad no sería necesario si hubiera

\footnotetext{
${ }^{29}$ O gobernanza moderna como es denominada por distintos autores (Rhodes 1997; Mayntz 1998; 2001; entre otros).
} 
un gobierno mundial. No obstante, actualmente los gobiernos de los estados tienen el monopolio legítimo del uso de la fuerza -el poder de ejecución. La gobernanza global por lo tanto, se refiere a la interacción política que se requiere para resolver los problemas que afectan a más de un estado o una región, cuando no hay poder para hacer cumplir, es decir, se refiere a la solución de problemas colectivos; no se refiere a la dominación. En la solución de ciertos problemas, la gobernanza global es dictada no sólo por intereses nacionales directos, sino también por un nuevo sentido de compromiso colectivo y de responsabilidad colectiva en torno a los acontecimientos globales (Mayntz 2002). La mejora de la solución global a los problemas no requiere, por supuesto, el establecimiento de instituciones formales globales más poderosas, sino que supondría la creación de un consenso sobre las normas y prácticas que deben aplicarse. Los regímenes internacionales constituyen, con mayor claridad y bastante abiertamente, soluciones transadas alcanzadas mediante negociación. Los pasos, por supuesto, para llevar a cabo el establecimiento de estas normas y que se están instaurando son la creación y mejora de los mecanismos de rendición de cuentas global. En este sentido, por ejemplo, el Pacto Mundial de Naciones Unidas ${ }^{30}$ - descrito como la más grande iniciativa voluntaria de responsabilidad corporativa del mundo - reúne a empresas, organismos nacionales e internacionales, sindicatos y otras organizaciones laborales y los distintos órganos de la sociedad civil para apoyar la protección universal del medioambiente, de los derechos humanos y de los principios sociales. La participación es totalmente voluntaria, y no hay vigencia de los principios por un organismo regulador exterior. Las empresas se adhieren a estas prácticas, tanto porque tienen sentido económico, como porque sus actores, incluyendo a sus accionistas (la mayoría individuos e inversores institucionales) se preocupan de estos temas y esto proporciona un mecanismo por el que se puede controlar fácilmente el cumplimiento de las empresas. No obstante, es sabido por todos, la existencia de relaciones asimétricas de poder en la gobernanza global; pero, como afirma Mayntz (2002: 7) la creciente regionalización, de la cual la Unión Europea es sólo la representante más avanzada (Comisión Europea 2001), obrará en contra de la perpetuación de las actuales asimetrías de poder y a favor del desarrollo de una estructura política verdaderamente global y de multinivel que permitirá mejorar la capacidad de los individuos y de las comunidades locales para que las empresas rindan cuentas.

\section{CONCLUSIONES}

Nuestro razonamiento en este trabajo es que la triple bottom-line es un concepto fácil que ha sido usado para dar a entender que la sostenibilidad y el desarrollo sostenible son objetivos sencillos de conseguir. Se ha argumentado (véase Aras \& Crowther 2009b;

\footnotetext{
${ }^{30}$ Ver http://www.unglobalcompact.org/languages/spanish/ consultado el 1 de abril de 2012.
} 
2008a) que la definición Brundtland del desarrollo sostenible es engañosa. En este trabajo se ha tratado de mostrar que las preocupaciones que son los requisitos previos de la sostenibilidad son diferentes de las implicaciones de la triple bottom line, hasta el punto de que representan una nueva triple bottom-line.

Figura 2.

La Triple Bottom-Line Real

\begin{tabular}{ccc}
\hline & Sostenibilidad & \\
\hline Performance & Performance & \\
Derechos humanos & Medioambiental & Gobernanza \\
\hline
\end{tabular}

Fuente: elaboración propia.

Nuestro argumento esencial es que no es posible considerar la sostenibilidad sin tener en cuenta la performance en cuestiones de derechos humanos, performance medioambiental y la gobernanza, tres factores que, juntos, representan un enfoque basado en la perspectiva del Contrato Social y que representa el aspecto de distribución de la durabilidad previamente identificado (ver Aras \& Crowther, 2009b). Como tales, proporcionan un mecanismo para centrarse en la toma de decisiones corporativas estratégicas. Más específicamente, y en el contexto de este trabajo, ellos hacen necesaria la inclusión de todas las personas en la preocupación social.

\section{REFERENCIAS BIBLIOGRÁFICAS}

Abberley, P. 1998. "Trabajo, utopía e insuficiencia". Pp. 76-96 en Discapacidad y sociedad. Madrid: Editorial Morata.

Amba-Rao, S. C. 1993. "Multinational Corporate Social Responsibility, Ethics, Interactions and Third World Governments: An Agenda for the 1990s". Journal of Business Ethics 12: 553-572.

Aras, G. y Crowther, D. 2008a. "Corporate sustainability reporting: a study in disingenuity?". Journal of Business Ethics 87 (supp 1): 279-288.

Aras, G. y Crowther, D. 2008b. "Governance and sustainability: An investigation into the relationship between corporate governance and corporate sustainability". Management Decision 46 (3): 433448.

Aras, G. y Crowther, D. 2008c. "The social obligation of corporations". Journal of Knowledge Globalisation 1 (1): 43-59.

Aras, G. y Crowther, D. 2008d. "Evaluating sustainability: a need for standards". International Journal of Social and Environmental Accounting 2 (1): 19-35. 
Aras, G. y Crowther, D. 2009a. "The Durable Corporation in a Time of Financial and Economic Crisis". Economics and Management 14: 211-217.

Aras, G. y Crowther, D. 2009b. The Durable Corporation: strategies for sustainable development. Aldershot: Gower.

Beder, S. 1997. Global Spin: The Corporate Assault on Environmentalism. Londres: Green Books.

Beurden, P. V. y Gössling, T. 2008. "The worth of values - a literature review on the relation between corporate social and financial performance". Journal of Business Ethics 82 (2): 407-424.

Bourdieu, P. 1984. Distinction: A Social Critique of the Judgement of Taste. Londres: Routledge \& Kegan Paul.

Bower, T. 2009. The Squeeze. Londres: Harper Press.

Brogna, P. 2006. "El nuevo paradigma de la discapacidad y el rol de los profesionales de la rehabilitación". Revista electrónica Cuadernos ESP 2 (2): 7-11. Consultado el 13 de mayo de 2010 (http:/l www.esp.ce.gov.br/cadernosesp/index.php/cadernosesp/article/view/17/15)

Brown, K., Tompkins, E. L. y Adger, W.N. 2002. Making Waves: integrating coastal conservation and development. Londres: Earthscan.

Chambers, R. 1994. "The Origins and Practice of Participatory Rural Appraisal." World Development 22 (7): 953-969.

Cohen, S., D. Demeritt, J. Robinson y D. Rothman. 1998. "Climate change and sustainable development: towards dialogue". Global Environmental Change 8:341-371.

Comisión Europea 2001. La Gobernanza Europea. Un Libro Blanco. Bruselas: Servicio de Publicaciones de la Comisión.

Crowther, D. 2002. A Social Critique of Corporate Reporting. Aldershot: Ashgate.

Crowther, D., Davies, M. y Cooper, S. 1998. "Evaluating corporate performance: a critique of Economic Value Added". Journal of Applied Accounting Research. 4 (3): 2-34.

Crowther, D. y Seifi, S. 2010. "The future of corporate social responsibility" en Empresas, Empresarios e Responsibilidade Social CES, Lisbon (forthcoming).

Crowther, D. y Seifi, S. 2012. "Plundering social capital: the rejection of a legacy". Revista Humanidades e Políticas Públicas (forthcoming).

Daly, H.E. 1992. "Allocation, distribution, and scale: towards an economics that is efficient, just, and sustainable". Ecological Economics 6 (3): 185-193.

Deffeyes, K.S. 2004. "Hubbert's Peak: The Impending World Oil Shortage". American Journal of Physics 72 (1): $126-127$.

Elliott, S.R. 2005. "Sustainability: an economic perspective." Resources Conservations and Recycling. 44: 263-277.

Ferreira, M. A. V. 2008. "Una aproximación sociológica a la discapacidad desde el modelo social: apuntes caracteriológicos". Revista Española de Investigaciones Sociológicas (124): 141-174.

Fukuyama, F. 1996. Trust: The Social Virtues and the Creation of Prosperity. New York: The Free Press. 
Gray, R. 1992. "Accounting and environmentalism: an exploration of the challenge of gently accounting for accountability, transparency and sustainability". Accounting, Organizations \& Society 17 (5): 399-425.

Gray, R. y Bebbington, J. 2001. Accounting for the Environment. Londres: Sage.

Hart, S.L. 1997. "Beyond greening: Strategies for a sustainable world". Harvard Business Review 75 (1): 66-76.

Hart, S.L. y Milstein, M.B. 2003. "Creating sustainable value". Academy of Management Executive 17 (2): 56-67.

Herremans, I.M., Akathaparn, P. y McInnes, M. 1992. "An investigation of corporate social responsibility, reputation and economic performance". Accounting, Organizations \& Society 18 (7/8): 587-604.

Hobbes, T. 1651. Leviathan. Many editions.

Hudson, L.J. 2009. The Enabling State: Collaborating for Success. Londres: Foreign and Commonwealth Office.

Human Rights Council 2009. "Promotion of all Human Rights, Civil, Political, Economic, Social and Cultural Rights Including the Right to Development". Nueva York: ONU.

Instituto Nacional de Estadística 2010. "Encuesta de Discapacidad, Autonomía personal y situaciones de Dependencia (EDAD2008)". Consultado el 13 de junio de 2012 (http://www.ine.es/jaxi/menu. do?type=pcaxis\&path=\%2Ft15/p418\&file=inebase\&L=0).

Ladkin, A. y Bertramini, A.M. 2002. "Collaborative Tourism Planning: a case study of Cusco, Peru". Current Issues in Tourism 5 (2): 71-93.

Lomborg, B. 2001. The Skeptical Environmentalist. Cambridge: Cambridge University Press.

Lovelock, J. 2006. The Revenge of Gaia. Harmondsworth: Penguin.

Marrewijk, M. van y Werre, M. 2003. "Multiple levels of corporate sustainability". Journal of Business Ethics $44(2 / 3):$ 107-119.

Marsden, C. 2000. "The new corporate citizenship of big business: part of the solution to sustainability". Business \& Society Review 105 (1): 9-25.

Mayntz, R. 1998. "New Challenges to Governance Theory". Jean Monet Chair Papers №50, European University Institute, Florence.

Mayntz, R. 2001. "El Estado y la sociedad civil en la gobernanza moderna". Revista del CLAD Reforma y Democracia (21): 1-8. Consultado el 1 de abril de 2012 (http://ensayoes.com/tw_files/31/d30889/7z-docs/14.pdf).

Mayntz, R. 2002. "Common Good and Governance" Pp. 15-27 en Common Good, Reinventing European and International Governace. New York/ Londres: Rowman and Littlefield.

Mishan, E.J. 1967. The Costs of Economic Growth. Harmondsworth: Pelican.

Moyano, E., A. Paniagua, y R. Lafuente 2009. "Políticas ambientales, cambio climático y opinión pública en escenarios regionales. El caso de Andalucía". Revista internacional de Sociología 67 (3): 681699 . 
Murray A., Haynes K. y Hudson L. J. 2010: "Collaborating to achieve corporate social responsibility and sustainability? Possibilities and problems". Sustainability Accounting, Management and Policy Journal 1 (2): 161-177.

Ormerod, P. 1994. The Death of Economics. Londres: Faber and Faber.

Pretty, J.N. 1995. "Participatory Learning for Sustainable Agriculture". World Development 23 (8): 1247-1263.

Reed, R. y DeFillippi, R.J. 1990. "Causal ambiguity, barriers to imitation, and sustainable competitive advantage". Academy of Management Review 15 (1): 88-102.

Rhodes, R. A. W. 1997. Understanding Governance. Policy Networks, Governance, Reflexivity and Accountability. Buckingham/Philadelphia: Open University Press

Rodríguez Díaz, S. y M. A. Ferreira 2010. "Desde la Dis-Capacidad hacia la diversidad funcional. Un ejercicio de Dis-Normalización". Revista Internacional de Sociología 68 (2): 289-309.

Rosenau, J.N. y Czempiel, E.O. 1992. Governance without Government. Cambridge: Cambridge University Press.

Rousseau, J.J. 1762. The Social Contract. Many editions.

Royal College of Engineering 2010. Global Water Security - an engineering approach. Londres: Royal College of Engineering.

Schmidheiny, S. 1992. Changing Course. New York: MIT Press.

Spangenberg, J.H. 2004. "Reconciling sustainability and growth: criteria, indicators, policies". Sustainable Development 12: 74-86.

Tinker, T. 1988. "Panglossian accounting theories: the science of apologising in style". Accounting, Organizations \& Society 13 (2): 165-189.

United Nations 2005. World Summit Outcome A/60/355. New York: UN.

United Nations 2009. "Promotion of all human rights, civil, political, economic, social and cultural rights, including the right to development A/HRC/11/13". Consultado el 10 de junio de 2012 (http://www2. ohchr.org/english/bodies/hrcouncil/docs/11session/A.HRC.11.13.pdf)

Williamson, O.E. 1975. Markets and Hierarchies: Analysis and Anti-trust Implications. New York: The Free Press.

World Commission on Environment and Development (WCED) 1987. Our Common Future (The Brundtland Report). Oxford: Oxford University Press.

Zwetsloot, G.I.J.M. 2003. "From management systems to corporate social responsibility". Journal of Business Ethics 44 (2/3): 201-207.

David Crowther es Catedrático de Responsabilidad Social Corporativa y Director del Centro de investigación en Gobernanza Organizacional, De Montfort University, UK. Director de Social Responsibility Journal y de una series de libros Gower sobre Responsabilidad Social Corporativa y de libros Emerald sobre desarrollo en Gobierno y Responsabilidad Corporativa. 
Teresa DuARTE-Atoche es Doctora por la Universidad de Sevilla (US) y profesora colaboradora en el Departamento de Contabilidad y Economía Financiera de la Universidad de Sevilla. Investigadora principal del proyecto de investigación de la US denominado "Impacto de las compañías andaluzas en la cohesión social"

RECIBIDO: 13/02/2013

ACEPTADO: 10/02/2014 